\title{
Application of Fuzzy Linear Optimization Techniques \\ to the Rural Roads Investment Resource Allocation Problem in the Offinso District of Ghana
}

\author{
Isaac F. Mensa-Bonsu, Seiichi Kagaya \\ and Etsuo Yamamura
}

\section{Introduction}

Many developing countries are now facing road maintenance crises with backlogs of maintenance needs. This present state of road deterioration has mainly been explained by the fact that, in the 1960's and 1970's road networks expanded faster than the corresponding maintenance budgets and institutional capacities. It has also been indicated that traffic has became heavier than expected, with axle loadings exceeding the designed capacity of roads $[14]$.

Although the problem of road deterioration is worldwide, it is perhaps more critical in sub-Saharan African countries where development efforts have been less successful and where, in particular, resource constraints are most severe. Given the constraints of scarce resources, vis-a-vis the massive road rehabilitation and improvement needs, both nationally and interntionally, greater efforts are now being made to develop and implement improved road planning and management tools. Road departments in all countries are under obligation to spend their budgets effectively.

One of the major problems confronting transportation planners and decision makers in developing countries, in fact, relates to optimally allocating the limited available budget to potential road candidates. The problem of developing analytical tools for road investment in general, and for rural roads investment in particular, in developing countries is compounded by issues of data, such as lack of socio-economic data on the zone of influence of the roads, high cost of data collection relative to project costs, inexactness of available data and the general fuzzy environment surrounding the decisions. Any mathematical models and techniques for assisting in resource allocation in developing countries should, therefore, take due consideration of these facts. Additionally, the mathematical sophistication of any such models and techniques should be considered against the background of comprehensibility [9].

In the light of the foregoing, this paper explores the use of Fuzzy Linear Optimization

Department of Regional Planning, Graduate School of Environmental Science, Hokkaido University, Sapporo, 060 JAPAN. 
techniques to the allocation of financial resources for rural roads improvement at the district level. Using as the basis, a Linear Programming model developed in an earlier study [7], this paper critically examines the practical realities of the fuzzy approach in a developing country, Ghana. We examine three main cases ;

i. Where the Decision Maker (DM) tolerates leeways in the budget limit and/or the aspiration level.

ii. Where the coefficients of the objective function and/or budget constraints are fuzzy numbers.

iii. Where the objective function is fuzzy.

Even though the budget and the policies for rural roads improvement are generally provided by the central government at the national level, much of the implementation decision-making takes place at the district or operational level. This paper, therefore, examines resource allocation for rural roads improvement at the district level. The models have been applied to the Offinso District in Ghana, a sub-Saharan African country.

Prior to the analysis a brief introduction is given to decision making in a fuzzy environment. This is followed by a brief discussion on the potential of fuzzy set theory in rural roads investment analysis in information-scarce developing countries.

\section{The Fuzzy Environment of Rural Roads Investment}

\subsection{Decision-Making in a Fuzzy Environment}

Decision-making in a fuzzy environment refers to a decision process in which the goals and/or the constraints, but not necessarily the system under control, are fuzzy in nature. That is, the goals and/or the constraints constitute classes of alternatives whose boundaries are not sharply defined [1]. Fuzzy goals and fuzzy constraints can be defined as fuzzy sets in the space of alternatives. A fuzzy decision is then defined as the fuzzy set of alternatives resulting from the intersection of the given fuzzy goals and the constraints. That is, given a fuzzy goal $G$ and a fuzzy constraint $C$ in the space of alternatives $X$, then $G$ and $C$ combine to form a decision $D$ resulting from the intersection of $G$ and $C$. This is expressed as $D=G \cap C$, where $\cap$ is the intersection operator. In terms of membership functions we have $\mu_{D}(x)=\mu_{G}(x) \wedge \mu_{C}(x)$ for each $x \in X$ where the minimum operator " $\wedge$ " is assumed, and where e.g. $\mu_{D}(x)$ is the membership function of the decision in the space of alternatives.

\subsection{The Potential of Fuzzy Set and Possibility Theory in Rural Road Planning and Management}

Recent advances in information systems, decision sciences, and computer technology can be utilized to improve rural roads planning and management in developing countries. Fuzzy Set mathematics and expert systems, mathematical programming techniques, and computer-aided techniques are some of the new tools and techniques which offer great potential in rural roads investment decision making. Sinha et al. [10], for instance, have examined some of these emerging tools and techniques, and their adaptability to state 
highway routine maintenance management.

The relevance of Fuzzy Set Theory to rural road planning is briefly discussed here. This relates basically to the kind of uncertainty involved in rural roads investment planning.

All interest in decision making processes are believed to stem from uncertainty. It is said that when we are certain what course of action is best, we simply perform it without further consideration. Whalen [11], for instance, provides an introduction to decision making under various kinds of uncertainty.

\subsubsection{Uncertainty}

Obstacles to certainty in decision making in general, and rural roads investment in particular, include:

a) Uncertainty about courses of action, due to lack of comprehension of all courses of action resulting from immensity of choice, imprecision of specifications, etc.

b) Uncertainty about consequences of each alternative course of action. This may be due to lack of knowledge about the possible future state of the world.

c) Uncertainty about preferences, due to a lack of knowledge of which set of consequences is preferable to any other achievable set.

Forms of uncertainty may include randomness, inexactness, ill-definedness and vagueness. It has been argued that while probability theory, including statistical decision analysis, Theory of Regret and Game Theory, can handle randomness, it cannot be applied to non-stochastic imprecision [1]. Also it cannot be applied when we have no exact information about relative likelihood of the outcome of each alternative, and when the outcomes are vaguely known in advance. Due to data issues, therefore, probability theory has limited application to rural roads investment decision making in developing countries.

Following the pioneering work of Zadeh, Fuzzy Set and Possibility Theory has been developed to handle the type of uncertainty which involves non-stochastic imprecision such as inexactness, ill-definedness and vagueness.

\subsubsection{Relevance of Fuzzy Set Theory to Rural Roads Investment Planning}

The specific issues giving credence to the use of Fuzzy Set Theory in rural roads investment analysis in developing countries include the following:

a) Net present value models usually used to support rural roads investment decisionmaking are based on the assumption that all data inputs can be uniquely determined. This can seldom be implied with regard to real life rural roads investment problems. Experience has shown that neither the project costs nor the benefits likely to accrue from rural road investments can be determined precisely [6].

b) Data on occurrence of events forms the backbone of probability theory. Lack of data on the occurrence of events in rural roads investment analysis limits the application of probability models. Fuzzy Set and Possibility Theory [15] may be applied in such situations. For instance, the probability that the improvement of a road link may lead to a certain kind of benefit may be difficult to estimate due to lack of data, but using Fuzzy Set Theory the possibility of that outcome may be estimated and utilized in decision 
making.

c) Most of the available data for rural roads investment planning are stated imprecisely. For instance, data on vehicle speeds on the roads are given in rough averages. Average vehicle speeds are generally given for an average vehicle. The average speed of a particular vehicle may be more or less than this given average vehicle speed. This situation may best be handled by fuzzy theory, where fuzzy numbers can be used.

Some of the data are also provided in ranges. In some cases respondents find it easier to give range data. The decision maker may also feel more comfortable in specifying vague data than point ones. Fuzzy Set Theory may be used to handle all of these situations.

d) The socio-economic situation in developing countries makes the application of rigid mathematical optimization techniques not very realistic. For instance, the budget to be made available for rural roads improvements in a particular district in a particular year may not be known with any certainty. In such cases, fuzzy mathematics provides a potential methodology.

e) Rural transport systems, of which roads form a part, are socio-economic systems characterized as 'soft' systems, compared to, say, machine systems, which are 'hard' technical systems. Fuzzy modeling as occurs in many 'soft' systems and human-computer systems can be viewed as yet another approach in rural transportation modeling.

In the next section we present a Crisp Linear Programming (LP) model for rural roads investment resource allocation which will form the basic model for studying the consequences of adopting fuzzy modeling approaches.

\section{Modeling Approach}

\subsection{The Resource Allocation Problem}

The resource allocation problem has been modeled for years using linear optimization or linear programming techniques. Linear optimization concerns the problem of making the best use of limited resources in relation to some criterion of value (see [3]). Hence it is well suited to the problem in hand, that is, the problem of optimally allocating the limited available budget for rural roads improvement.

We consider the main objective for rural roads improvement to be to increase rural accessibility. By rural accessibility is meant the ability of the villagers to reach or be reached by urban-based activities and services via the transport system. The accessibility of an individual, in a particular village, to a particular facility is a function of the travel time. The travel time depends on the road conditions (ceteris paribus) which may be measured by the average vehicle speed on the link considered.

Many rural roads are not passable the entire year. During seasons of impassability, the villagers may have to walk to facilities, and this should be reflected in the accessibility measure. Comparing the "with and without" situations, the increase in accessibility resulting from rural roads improvement may be obtained. The allocation model should also ensure that the increase in accessibility benefits the maximum number of villagers. 
The optimum resource allocation problem can be modeled as :

$$
\begin{gathered}
\max z=\sum_{i=1}^{N} \Delta \phi_{i}=\sum_{i=1}^{N} P_{i}\left[K_{1} x_{i}\left(\mathrm{~V}_{\text {iom }}\right)^{-1}+K_{2} x_{i}\left(V_{w}\right)^{-1}-365 x_{i}\left(\mathrm{~V}_{\text {iam }}\right)^{-1}\right] \\
\text { s.t. } \sum_{i=1}^{N} a_{i} x_{i} \leq b \\
o \leq x_{i} \leq u
\end{gathered}
$$

Where: $\Delta \phi_{i}=$ increase in accessibility for population $P_{i}$ accessed by link $i$.

$K_{1}, K_{2}=$ number of passable and impassable days, respectively, in a year

$V_{i o m}=$ average vehicle speed, without road improvement (in $\mathrm{km} / \mathrm{hr}$ )

$V_{\text {iam }}=$ average vehicle speed, with road improvement (in $\mathrm{km} / \mathrm{hr}$ )

$V_{w}=$ average walking speed (in $\mathrm{km} / \mathrm{hr}$ )

$a_{i}=$ average cost of improving $1 \mathrm{~km}$ of road

$b=$ available budget (financial resources) to be allocated.

$x_{i}=$ the length of link to be improved

$u=$ upper limit of $x_{i}$

Details of this approach can be found in [7]

\subsection{Reality of the Situation}

In reality, using the resource allocation model in (1)-(3) one may encounter uncertainty problems, of non-stochastic nature, due to the following real situations:

I) The Decision Maker (DM) tolerates leeways in the goal and/or budget constraints. For instance

a) The DM has an aspiration level or target accessibility increase to be strictly accomplished, but is prepared to tolerate violations of the budget provided for road improvements to some extent.

b) The DM is prepared to tolerate violation of his target accessibility increase within a certain limit but will not allow violations of the budget constraint.

II) The DM does not know exactly the values of the parameters or coefficients taking part in the problem. For instance, these might be the number of passable and impassable days, average vehicle speed, cost of road improvement, budget allocation, and so on.

III) The DM is not able to determine the exact relationship between rural road improvement and accessibility increase. That is the function which determines increase in accessibility resulting from rural roads improvement is imprecise due to a weak relationship between rural road improvement and accessibility increase, as defined in our problem.

This study examines the foregoing cases separately in order to observe any changes in the set of road links composing the solution set with changing situations.

Approaches to solving the resource allocation problems in the above cases are discussed in the subsequent section.

\subsubsection{Case I}

The type of Fuzzy Linear Optimization that is appropriate for Case I is what has been 
referred to as Flexible Programming [5]. The solution method is primarily based on Bellman-Zadeh's [1] method to decision-making in a fuzzy environment, briefly described in section 2.

Here we will use Zimmermann's [16] approach. The objective function is considered a fuzzy set characterized by its membership function, and so are the constraints. The fuzzy 'decision', therefore, is defined as the intersection of the fuzzy sets describing the constraints and the objective function. Using Zimmerman's approach, the maximizing decision can be defined as the solution with the highest degree of membership in the fuzzy 'decision set'. This approach is summarized below:

Mathematically, the original LP is expressed as a symmetrical model of the form:

find $\mathrm{x}$ such that

$$
\left.\begin{array}{r}
C^{T} x \geqq Z \\
A x \lesssim b \\
x \geq 0
\end{array}\right\}
$$

where $c$ and $x$ are n-vectors, $b$ is an $m$ vector, and $A$ is an $m x n$ matrix.

Here, "ミ" denotes the fuzzified version of $\leq$ with the linguistic interpretation "essentially smaller than or equal to".

$$
\begin{aligned}
& \text { Substituting } B=\left(\begin{array}{l}
-C^{T} \\
A
\end{array}\right) \text { and } d=\left(\begin{array}{l}
-Z \\
b
\end{array}\right) \text { (4) becomes } \\
& \left.\begin{array}{r}
B x \leqq d \\
x \geq 0
\end{array}\right\}
\end{aligned}
$$

the membership function of which are $\mu_{i}(x), i=1, \ldots, m+1$

The membership function of the fuzzy set decision of model (5) is given as

$$
\mu_{\mathrm{D}} \tilde{\mathrm{D}}(x)=\min _{i=1}^{m+1} \mu_{i}(x)
$$

The maximizing solution to (6) is expressed as

$$
\max _{x \geq 0} \min _{i=1}^{m+1} \mu_{i}(x)=\mu_{m}\left(x^{\circ}\right)
$$

where $x^{\circ}$ is a crisp optimal solution.

The membership function $\mu_{i}(x)$ is 0 if the constraints, including the objective function are strongly violated, 1 if they are very well satisfied, and increases monotoneously from 0 to 1 .

If $\mu_{i}(x)$ is assumed to be linearly increasing over a "tolerance interval" $\left[d_{i}, d_{i}+q_{i}\right]$ where $q_{i}$ are subjectively chosen constants of admissible violations of the constraints and the objective function, the maximizing solution (7) then becomes

$$
\max _{x \geq 0} \min _{i=1}^{m+1}\left\{1-\frac{(B x)_{i}-d_{i}}{q_{i}}\right\}
$$

Solution to (8) can be obtained by solving the following crisp LP : 


$$
\left.\begin{array}{ll}
\max & \lambda \\
\text { s.t. } & \lambda q_{i}+(B x)_{i} \leq d_{i}+q_{i} \\
& 0 \leq \lambda \leq 1 \\
& x \geq 0
\end{array}\right\}
$$

where $\lambda$ is the variable that indicates the value of the minimum membership function of the fuzzy decision set. If the optimal solution to (9) is the vector $\left(\lambda^{\circ}, x^{\circ}\right)$ then $x^{\circ}$ is the maximizing solution of (7).

Applying this approach to Case I, the road selection can be obtained by solving the following crisp LP.

Ia $\max \lambda$

$$
\left.\begin{array}{ll}
\text { s.t. } & \sum_{i=1}^{N} P_{i}\left[K_{1} x_{i}\left(\mathrm{~V}_{i o m}\right)^{-1}+K_{2} x_{i}\left(V_{w}\right)^{-1}-365 x_{i}\left(\mathrm{~V}_{i a m}\right)^{-1}\right] \geq Z^{0} \\
& \lambda q+\sum_{i=1}^{N} a_{i} x_{i} \leq b+q \\
& 0 \leq x_{i} \leq u, \lambda \geq 0
\end{array}\right\}
$$

where $z^{0}$ is the target accessibility increase

IIb. $\max \lambda$

$$
\left.\begin{array}{ll}
\text { s.t. } & \lambda q+\sum_{i=1}^{N} P_{i}\left[K_{1} x_{i}\left(\mathrm{~V}_{i o m}\right)^{-1}+K_{2} x_{i}\left(V_{w}\right)^{-1}-365 x_{i}\left(\mathrm{~V}_{i a m}\right)^{-1}\right] \geq Z_{0}+q \\
& \sum_{i=1}^{N} a_{i} x_{i} \leq b \\
& 0 \leq x_{i} \leq u \\
\lambda \geq 0 & \lambda \geq
\end{array}\right\}
$$

\subsubsection{Case II}

Case II can be modeled as an FLP with fuzzy coefficients. Here, since the DM cannot specify the parameters exactly, for instance, the exact number of passable days, cost of road improvement, or average vehicle speed, we shall represent them by fuzzy numbers. Since the product of two fuzzy numbers also yields a fuzzy number based on approximation formula (see [2]), the objective function coefficients in our original allocation model (1)-(3) can be represented by single fuzzy numbers. We assume triangular fuzzy numbers, where a triangular fuzzy number is a special type of the so-called L-R fuzzy number (see e.g. [2], [13] or [16]).

Triangular fuzzy numbers are characterized by membership functions of the form :

$$
\mu_{A}(x)= \begin{cases}L(x) & \text { for }-\infty \leq \underline{a} \leq x \leq a \\ R(x) & \text { for } \quad a \leq x \leq \bar{a} \leq+\infty \\ 0 & \text { otherwise }\end{cases}
$$

where $L$ is a nondecreasing linear function, and $R$ is a nonincreasing linear function such that $L(a)=R(a)=1 ; \quad L(\underline{a})=R(\bar{a})=0$.

The value $a$ is referred to as the mean value of $\mathrm{A} ; \underline{a}$ and $\bar{a}$ are the lower and upper bounds, respectively, of $A$. Symbolically, a triangular fuzzy number may be denoted as 
$(\underline{a}, a, \bar{a})$, and depicted as in Figure 1 .

Here, we use Wierzchon, Kindler and Tyszewski's approach (we will call it WKT approach) as presented in [13]. There are other approaches such as Tanaka and Asai's approach [11].

The WKT approach also makes reference to Bellman-Zadeh's rule, and takes the form :

$$
\left.\begin{array}{l}
\max \alpha \\
v\left(h_{k}(x), B_{k}\right) \geq \alpha, k=1,2, \ldots, N \\
x \geq 0 ; \alpha>0
\end{array}\right\}
$$

$N=$ number of constraints + number of objective functions.

where : $h_{k}(x)$ is composed of the LHS's (left hand side) of the objective and constraint functions, similar to $B_{x}$ in (5); and $B_{k}$ is composed of the RHS's of the objective and constraint functions, similar to $\mathrm{d}$ in (5).

In [13] a fuzzy function is defined as a mapping $f: Y \rightarrow F(\boldsymbol{R})$ assigning fuzzy numbers to the points in $Y$. From a mathematical standpoint, treating the fuzzy mapping as a generalized multivalued mapping implies that to determine a co-image of a fuzzy set $A$ we must specify two sets called the upper $\left(A^{*}\right)$ and lower $\left(A_{*}\right)$ inverses of $\mathrm{A}$, defined as :

$$
\mu_{A}^{*}(y)=\operatorname{Inter}(Z, A)=\sup _{u \in R} \min \left(\mu_{z}(u), \mu_{A}(u)\right)
$$

and

$$
\mu_{A *}(y)=\operatorname{Incl}(Z, A)=\inf _{\mathrm{u} \in R} \max \left(1-\mu_{z}(u), \mu_{A}(u)\right)
$$

where $Z=f(y)$, Inter $(Z, A)$ is the degree of intersection of $Z$ with $A$ and $\operatorname{Incl}(Z, A)$ is the degree of inclusion of $Z$ in $A$.

From (13) the DM can construct a set of admissible alternatives by defining :

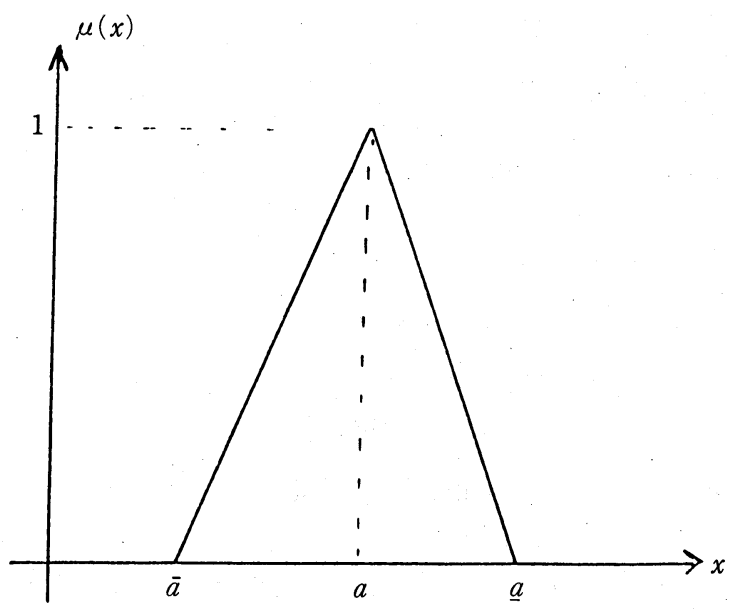

Fig. 1 A triangular fuzzy number 


$$
X_{k}^{v}(\alpha)=\left\{x \in X: v\left(h_{k}(x), B_{k}\right) \geq \alpha\right\}
$$

to be the set of alternatives satisfying the $k_{t h}$ criterion (objective function or constraint) with the degree not less than $\alpha$. Here, $v \in\left\{\right.$ Inter, Incl, Incl $_{1}$ )

Incl $_{1}$ has been defined as (see [13])

$$
\operatorname{Incl}_{1}(Z, A)=\left\{\begin{array}{l}
1 \text { when } Z \text { is included in } A \text { in the 'hard' sense }\left(i e Z \subseteq{ }_{h} A\right) \\
\alpha \in(0,1) \text { When Supp } A \subseteq \operatorname{Supp} Z \\
0 \quad \text { otherwise }
\end{array}\right.
$$

where : supp A represents the support of the fuzzy set $A$.

In this study we consider two cases where:

i) $v=$ inter and ii) $v=\operatorname{Incl}_{1}$.

Considering all the parameters specified in our resource allocation model (1)-(3) as triangular fuzzy numbers, and using $v=$ Inter index, we need to solve the following crisp equivalent of (13);

$$
\left.\begin{array}{c}
\max \alpha \\
\text { s.t. }(\overline{\boldsymbol{c}}-\alpha(\overline{\boldsymbol{c}}-\boldsymbol{c})) \boldsymbol{x} \geq Z^{-}+\alpha\left(Z^{+}-Z^{-}\right) \\
\quad \underline{\boldsymbol{a}}+\alpha(\boldsymbol{a}-\underline{\boldsymbol{a}})) \boldsymbol{x} \leq b^{+}-\alpha\left(b^{+}-b^{-}\right) \\
\quad \boldsymbol{x} \geq \mathbf{0}, \alpha>0
\end{array}\right\}
$$

Where $x$ is an $(\mathrm{M} \times 1)$ column vector,

$C$ and $\boldsymbol{a}$ are the $(1 \times \mathrm{M})$ row vectors

$Z$ and $b$ are real numbers

Using $v=\operatorname{Incl}_{1}$, we have

$$
\left.\begin{array}{c}
\max \alpha \\
\text { s.t. }(\underline{\boldsymbol{c}}+\alpha(\boldsymbol{c}-\underline{\boldsymbol{c}})) \boldsymbol{x} \geq Z^{-}+\alpha\left(Z^{+}-Z^{-}\right) \\
\left(\overline{\boldsymbol{a}}-\alpha(\overline{\boldsymbol{a}}-\boldsymbol{a}) \boldsymbol{x} \leq b^{+}+\alpha\left(b^{+}-b^{-}\right)\right. \\
\underline{\boldsymbol{c} \boldsymbol{x} \geq Z^{-}} \\
\quad \overline{\boldsymbol{a}} \boldsymbol{x} b^{+} \\
\quad \boldsymbol{x} \geq 0, \alpha \in[0,1]
\end{array}\right\}
$$

The interpretation is that since Incl $\left(h_{k}(x), B_{k}\right)$ is not greater than Inter $\left(h_{k}(x), B_{k}\right)$, the road selection based on Incl index will be more acceptable than those obtained by the Inter Index. In the Inter index the DM prefers a risky strategy, while when using Incl index the DM prefers a careful strategy. The $\mathrm{Incl}_{1}$ index is used instead of Incl in the case of a very important criterion where the $\mathrm{DM}$ is interested in considering alternatives for which the support of the fuzzy number $h_{k}(x)$ is surely contained in the support of the satisfiability region $\left(B_{k}\right)$

\subsubsection{Case III}

Case III can be modeled as an FLP of the form 


$$
\begin{aligned}
& \max \tilde{g}_{j}(x), j=1, \ldots, J \\
& \text { s.t. } x \in X
\end{aligned}
$$

Where $\tilde{g}$ is a fuzzy function and $X$ is a crisp set of admissible alternatives. We adopt Wierzchon's approach which is also based on the classical theory of choice.

Two types of solutions to (19) are considered in this study referred to as the $\alpha$-Internondominated alternative and the $\alpha$-inter -dominating alternative (see [13]) for details).

Representing the left and right reference functions of the fuzzy number $\widetilde{g}(x)$ as $L_{\tilde{g}(x)}$ and $R_{\tilde{g}(x)}$ respectively, (19) reduces to the following mathematical programming problems :

$$
\left.\begin{array}{ll}
\max & R_{\tilde{g}(x)}^{-1}(\alpha) \\
\text { s.t. } & x \in X, 0<\alpha<1
\end{array}\right\}
$$

and

$$
\left.\begin{array}{ll}
\max & L_{\tilde{g}(x)}^{-1}(\alpha) \\
\text { s.t. } & x \in X, 0<\alpha<1
\end{array}\right\}
$$

When $\tilde{g}(x)=\mathrm{cx}$ and all the components of $\mathrm{c}$ are triangular fuzzy numbers, then the $\alpha$-Inter-nondominated alternative can be obtained by solving :

$$
\left.\begin{array}{l}
\max (\bar{c}-\alpha(\bar{c}-c)) x \\
\text { s.t. } x \in X, 0<\alpha \leq 1
\end{array}\right\}
$$

also the $\alpha$-Inter-dominating alternative is obtained from :

$$
\left.\begin{array}{l}
\max (\underline{c}+\alpha(c-\underline{c})) x \\
\text { s.t. } x \in X, 0<\alpha \leq 1
\end{array}\right\}
$$

These two approaches have been applied to case III.

\section{Application to the Offinso District in Ghana}

The methods discussed so far will be applied to Offinso District of Ghana to allocate a budget of 120 million Cedis for rural roads improvement in a particular year. The relevant features of the district are indicated in Table 1 (see also Figure 2).

The district is traversed more or less centrally by a highway, along which virtually all the facilities in the district are located and about 67 per cent of the population live. About 26,546 persons live off the highway and are connected to the highway by a network of feeder roads and tracks. These rural roads have deteriorated tremendously causing accessibility problems to the off-highway villagers.

The cost of improving 1 kilometer of feeder road to a gravel standard is taken as 2 million Cedis and that of upgrading a track 3.5 million Cedis. For the purpose of the analyses the feeder roads and tracks have been divided into 38 links and 39 links, respectively. The basic data required for the analyses are indicated in the resource allocation model in (1)-(3). The population accessed by each link, $\mathrm{P}_{i}$, is obtained as the cumulative 
Table 1 Relevant features of offinso district

\begin{tabular}{l|l}
\hline \hline Area & $1,540 \mathrm{sq} . \mathrm{km}$. \\
Highway & $73 \mathrm{~km}$. \\
Feeder Roads (38 links) & $135.7 \mathrm{~km}$. \\
Tracks (39 links) & $101.8 \mathrm{~km}$. \\
Population & 81,345 \\
$\quad$ along highway & 54,799 \\
$\quad$ off highway & 26.546 \\
Average vehicle speeds & \\
$\quad$ Feeder roads & $20 \mathrm{~km} / \mathrm{h}$ \\
$\quad$ Tracks & $15 \mathrm{~km} / \mathrm{h}$ \\
Passable days (average) & \\
$\quad$ Feeder roads & 350 \\
$\quad$ Tracks & 275 \\
\hline
\end{tabular}

number of villagers who need the link in order to acquire access to the highway (refer to Figure 2). The population data cannot be provided due to space limitations. The parameters $K_{1}, K_{2}$, and $V_{\text {iom }}$ are found in Table 1 . Average walking speed is taken to be $4 \mathrm{~km} / \mathrm{hr}$ and average vehicle speed after road improvement is also taken as $60 \mathrm{~km} / \mathrm{hr}$.

Substituting the values of the parameters into our crisp model in (1)-(3), for instance, the district rural roads investment resource allocation model becomes:

$$
\left.\begin{array}{c}
\max Z=\sum_{i=1}^{38} 15.2 P_{i} \chi_{i}+\sum_{j=1}^{39} 34.75 P_{j} \chi_{j} \\
\text { s.t. } \quad 0 \leq \sum_{i=1}^{38} 2 \chi_{i}+\sum_{j=1}^{39} 3.5 \chi_{j} \leq 120 \\
0 \leq \chi_{i}, \chi_{j} \leq U_{j, j}
\end{array}\right\}
$$

where $i$ relates to feeder roads $j$ relates to tracks.

\section{Summary of results and Their Implications}

Applying the crisp model in (1)-(3) to the Offinso District yields $z=2,154,824$ and the optimal roads selection indicated in Figure 2. $Z$ is measured in man-hours and indicates the maximum increase in accessibility resulting from the roads improvement, as measured by time savings.

The results of the fuzzy mathematical analysis of the crisp model are presented below

\subsection{Case I}

Case I was further investigated, with the results indicated in Table 2.

In Case Ia, the set of roads selected for improvement remain the same as in the case of the crisp LP. The degree of membership in the fuzzy set decision is about 1, indicating that the constraints can be satisfied in the crisp sense, without violations. It also shows that the decision maker can be completely satisfied. 


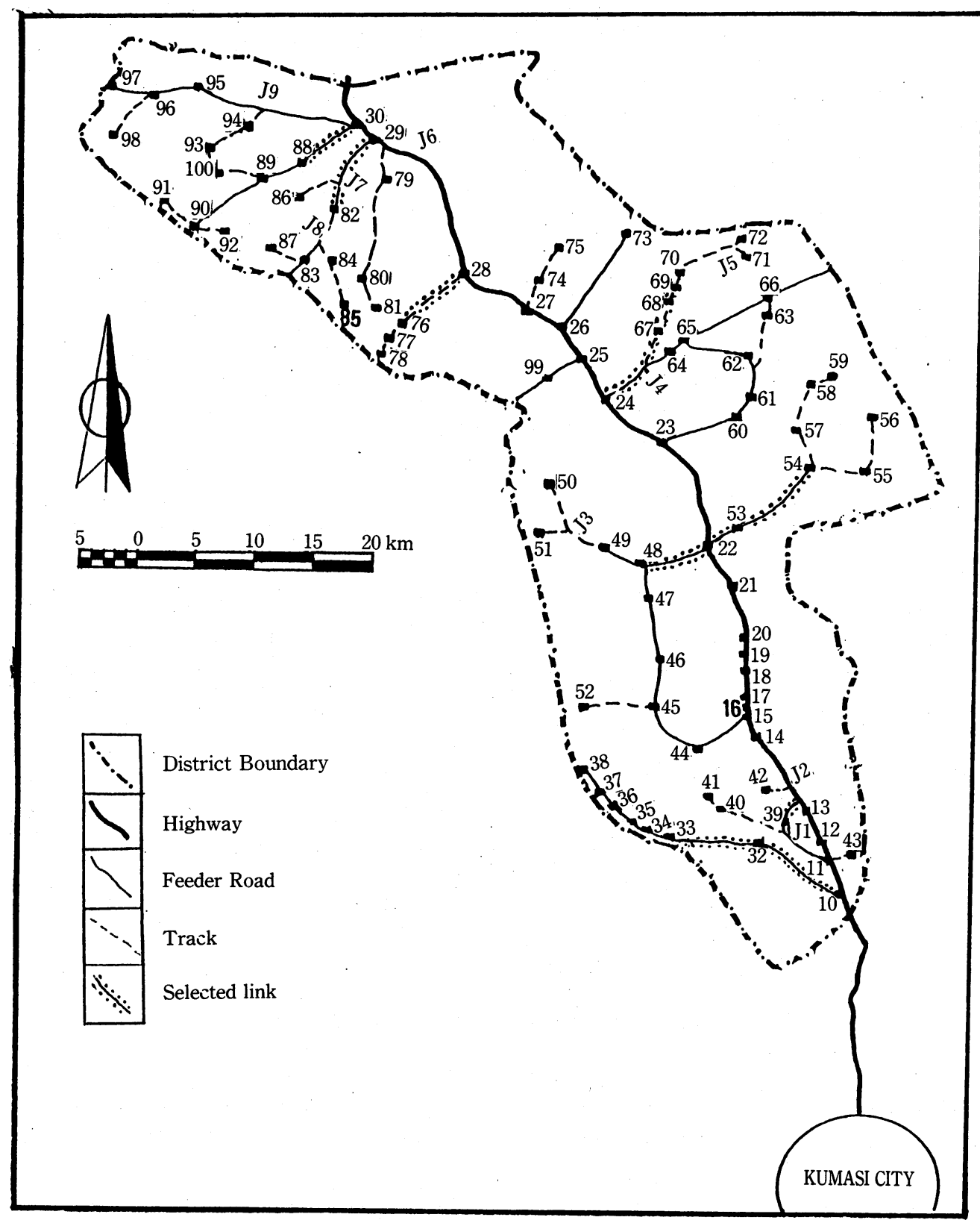

Fig. 2 Optimal selection of roads using Crisp LP

The results of Case $1 \mathrm{~b}$ are shown in Figure 3.

In each case of the budget constraint considered, the decision maker cannot be completely satisfied because of violations of budget albeit within a tolerable limit. When the budget increases, the degree of satisfaction also increases. For the same budget, when admissible violations are increased, the degree of satisfaction also increases, rapidly at first 
Table 2 Results of further investigation of case I

\begin{tabular}{c|c|c|c|c|c|c}
\hline \hline Case & $\begin{array}{c}\text { Aspiration } \\
\text { Level }\end{array}$ & $\begin{array}{c}\text { Violation } \\
\text { Tolerable }\end{array}$ & Budget & $\begin{array}{c}\text { Violation } \\
\text { Tolerable }\end{array}$ & $\begin{array}{c}\text { Change } \\
\text { in Roads }\end{array}$ & $\begin{array}{c}\text { Satisfacti- } \\
\text { on index }\end{array}$ \\
\hline $1 \mathrm{a}$ & $2,154,800$ & No & $120 \mathrm{~m}$ & Yes & No & 1.0 \\
$1 \mathrm{~b}$ & $2,154,800$ & No & Reduced & Yes & No & $<1.0$ \\
$1 \mathrm{c}$ & $2,154,800$ & No & Increase & Yes & Yes & 1.0 \\
$1 \mathrm{~d}$ & $2,154,800$ & Yes & $120 \mathrm{~m}$ & No & No & $<1.0$ \\
$1 \mathrm{l}$ & Reduced & Yes & $120 \mathrm{~m}$ & No & Yes & 1.0 \\
\hline
\end{tabular}

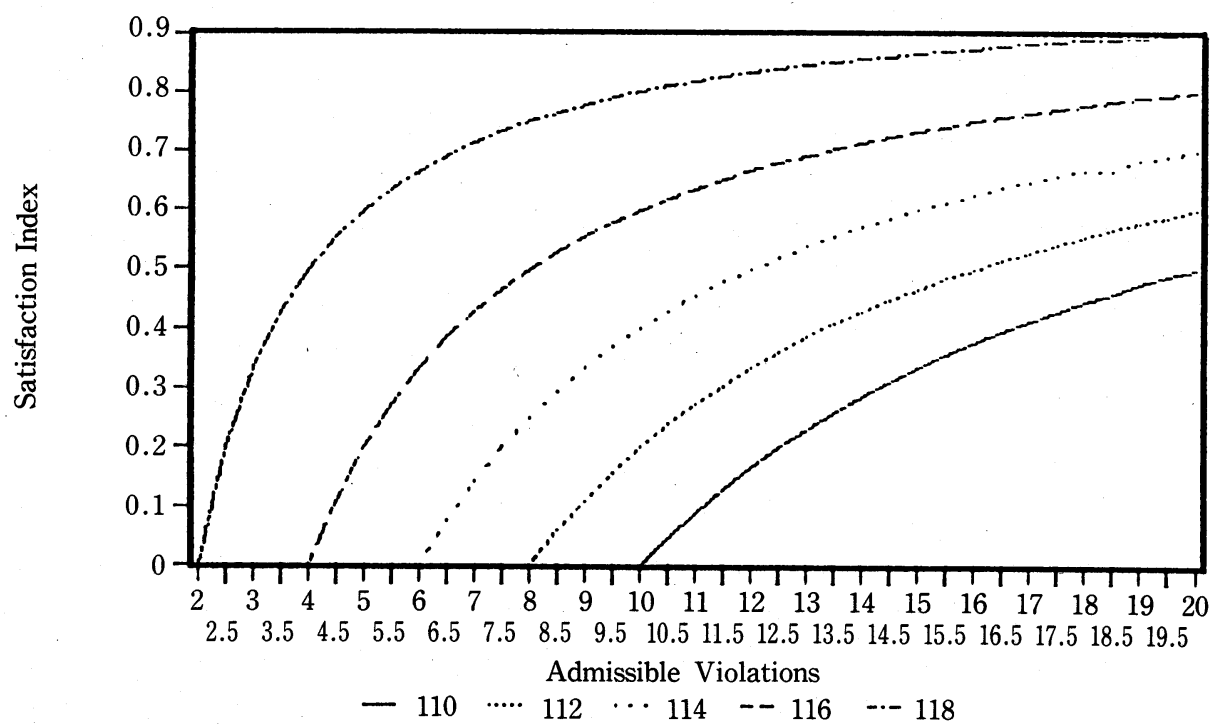

Fig. 3 Changes in degree of satisfaction with respect to changes in admissible violations of budget constraint

but less than proportionately after some time. For example, for a budget of 118 million Cedis the degree of satisfaction increased slowly after an admissible violation of 7 million Cedis.

There is no change in roads selected for improvement compared with the crisp case.

In Case 1c, there are some changes in the roads selected. For example, when the budget is increased to 125 million Cedis with an admissible violation of 10 million Cedis, feeder road links 88-30 and 33-32 are dropped and instead track links 77-76, 70-69, 55-54, 43-11 are selected. There is complete satisfaction with this selection.

The results for Case 1d are depicted in Figure 4.

There is no change in road selection compared with the crisp LP case. The decision maker's degree of satisfaction increases as the tolerance interval is increased. From Figure 4 it can be seen that after a tolerance level of 2,500 the degree of satisfaction increases slowly. A tolerance interval of 2,500 or 3,000 could be recommended in such a situation. 


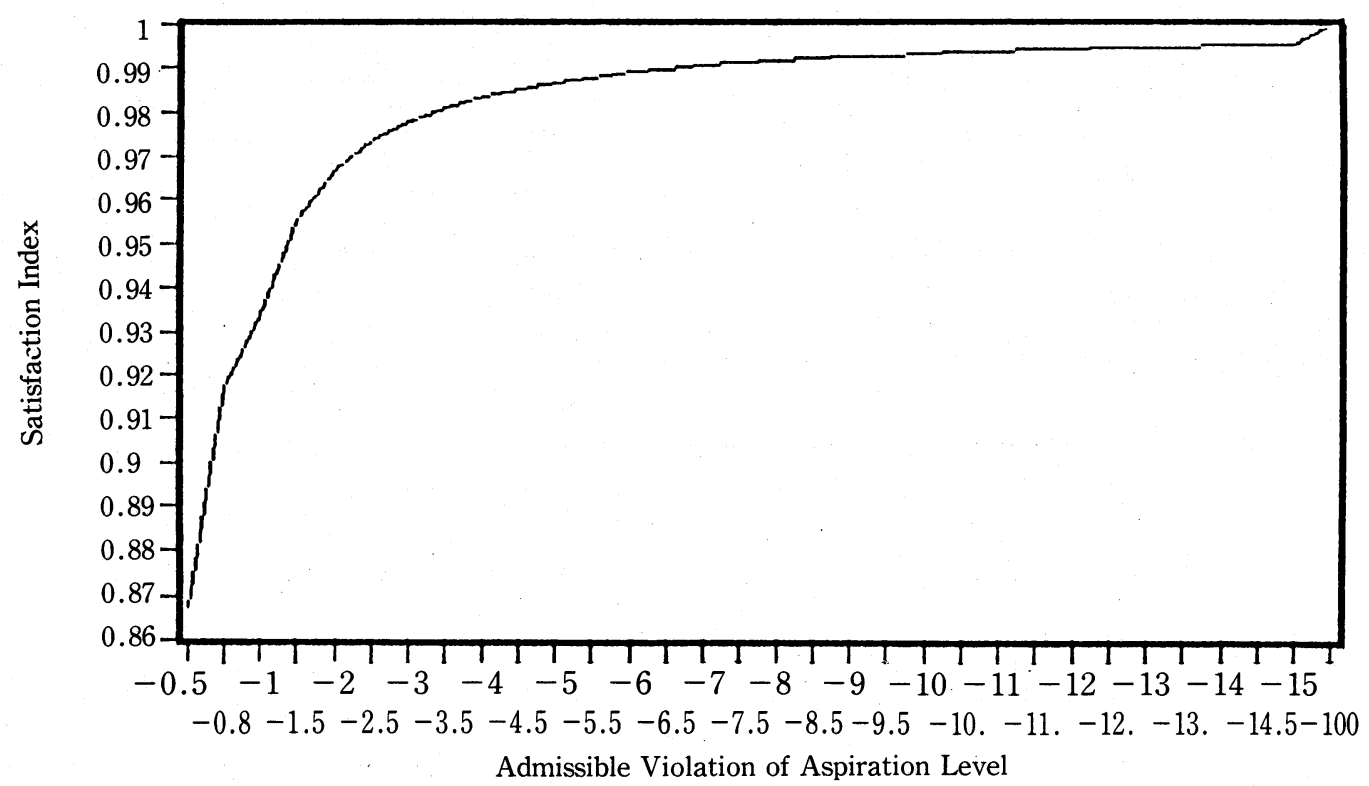

Fig. 4 Changes in degree of satisfaction with respect to changes in admissible violations of aspiration level

In case 1e, it is observed that when aspiration level is reduced slightly, say to $2,153,800$ with a tolerance interval of 500 , there is no significant change in the set of roads selected. When it is reduced by a good margin, say to $2,110,800$ with a tolerance interval of 500 , significant changes occur. For example, feeder road links 33-32 and 88-30 are dropped as (in Case 1c) in favor of track links 55-54 and 77-76.

\subsubsection{Degree of Violation of Aspiration Level}

Table 3 indicates the degree of violation of the aspiration level when the available budget is lower than in the case of the crisp LP.

Given a budget of 118 million Cedis, for instance, the aspiration level will be violated by a margin of about $23,986.33$ or about 1.1 per cent.

This could be interpreted to mean that for a budget of 118 million Cedis, the aspiration level of $2,154,800$ will be short of about 1.1 per cent. It could also mean that the decision

Table 3 Degree of violation of aspiration level with decreased budget

\begin{tabular}{c|c|c|c|c}
\hline \hline Budget & Aspiration & Tolerable Limit & Violation & Satisfaction \\
\hline $118 \mathrm{~m}$ & $2,154,800$ & 30,000 & $23,986.33$ & 0.20045 \\
$116 \mathrm{~m}$ & $2,154,800$ & 50,000 & $47,906.33$ & 0.041873 \\
$114 \mathrm{~m}$ & $2,154,800$ & 80,000 & $72,334.34$ & 0.095824 \\
$112 \mathrm{~m}$ & $2,154,800$ & 100,000 & $97,524.33$ & 0.024757 \\
$110 \mathrm{~m}$ & $2,154,800$ & 130,000 & $122,714.3$ & 0.056044 \\
\hline
\end{tabular}


maker needs to reduce his aspiration level by about 1.1 per cent.

\subsection{Case II}

In obtaining the triangular fuzzy numbers for the analysis we considered the coefficients and values in the crisp case as the mean values and the $L$ and $R$ references taken as $\pm 5 \%$ of the mean value, e.g. $\underline{b}=0.95 b ; \bar{b}=1.05 b$

Two fuzzy intervals were used for the analysis as follows :

For objective function value:
a) $\left.\begin{array}{r}Z^{-}=Z \\ Z^{+}=\bar{Z}\end{array}\right\}$ where the interval taken is $(\tilde{Z},+\infty)$
b) $\left.\begin{array}{l}Z^{-}=\underline{Z} \\ Z^{+}=Z\end{array}\right\}$ where the fuzzy interval taken is $[\tilde{Z},+\infty)$

For budget constraint :
a) $\left.\begin{array}{l}b^{-}=\underline{b} \\ b^{+}=b\end{array}\right\}$ where the fuzzy interval taken is $(-\infty, \tilde{b})$
b) $\left.\begin{array}{l}b^{-}=b \\ b^{+}=\bar{b}\end{array}\right\}$ where the fuzzy interval taken is $(-\infty, \tilde{b}]$

The fuzzy interval $(\tilde{z},+\infty)$ is interpreted as the fuzzy interval of numbers certainly greater than $\tilde{z}$ and the interval $[\tilde{z},+\infty)$ as numbers possibly not less than $\tilde{z}$.

Case II was, therefore further divided into Case IIa and Case IIb to illustrate just two of the possible analyses.

In case IIa, we assume that:

i. The accessibility increase from the road improvement will certainly be greater than 2,154,800 man-hours (the objective function value obtained in (1)-(3)).

ii. The available budget for road improvement in the district will be possibly not less than 120 million Cedis.

In Case IIb, we assume that:

1. The accessibility increase will be possibly not less than $2,154,800$ man-hours

ii. The budget will certainly be greater than 120 million Cedis.

These two cases were examined using the Inter index and the Incl $_{1}$ index. The Inter index may perhaps represent an optimistic case, based on the assumption that:

a) the actual increase in accessibility resulting from improving each link will certainly be higher than the estimated values;

b) the cost of improvement may be lower than expected.

The Incl $_{1}$ index may also represent a pessimistic case, which may be based on the assumption that:

a) the actual increase in accessibility resulting from improving each link may be less than the estimated values; 


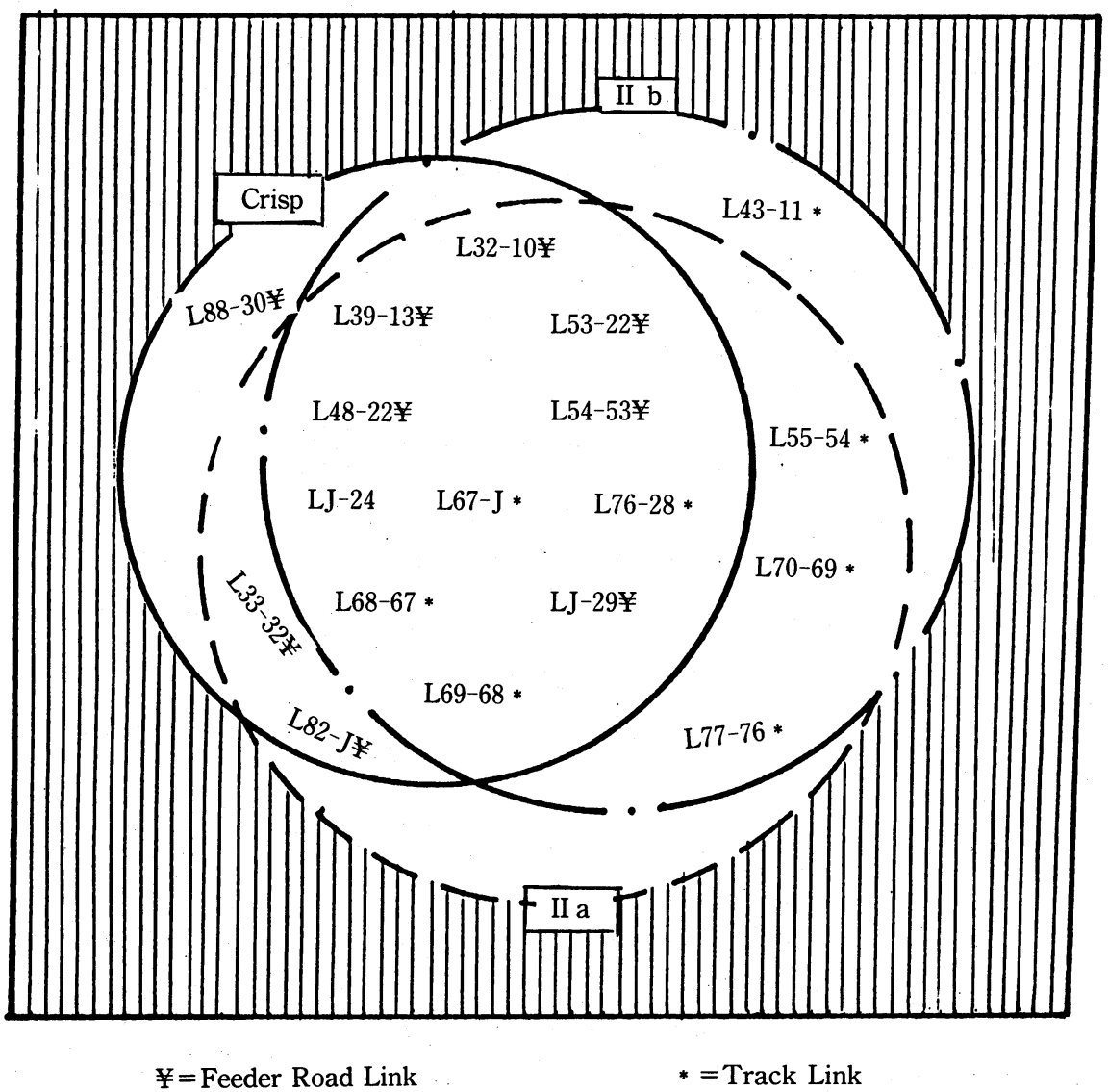

Fig. 5. Selection of roads in Case II based on Inter index relative to selection in crisp LP case

b) the.cost of improvement may actually be higher than expected.

Assuming the DM prefers a risky strategy, then the Inter index is used; where DM wants a very careful strategy $\operatorname{Incl}_{1}$ is used. When DM prefers a very careful strategy then given case II, no feasible solution can be obtained. The selection using the Inter index is indicated in Figure 5. The feasibility of solution in cases IIa and IIb using Inter index can also be seen in Table 4. Many more analyses can be carried out relating to case II by changing the assumptions. This can give the DM a greater knowledge about the invest-

Table 4 Feasibility of solution in cases IIa and IIb using inter index

\begin{tabular}{c|c|c|c|c|c|c}
\hline \hline & \multicolumn{7}{|c}{ Degree of Satisfaction of DM } \\
\hline Case & 0.0 & 0.2 & 0.4 & 0.6 & 0.8 & 1.0 \\
\hline IIa & Yes & Yes & Yes & No & No & No \\
IIb & Yes & Yes & Yes & Yes & Yes & No \\
\hline
\end{tabular}


ment decision.

\subsection{Case III}

The results obtained using $\alpha$-inter-nondominated (22), indicates the solution with the maximal degree of membership in the feasibility set. $\alpha$-inter-dominating yields the road selection with the minimal degree of membership. This can be seen in Figure 6 , were a

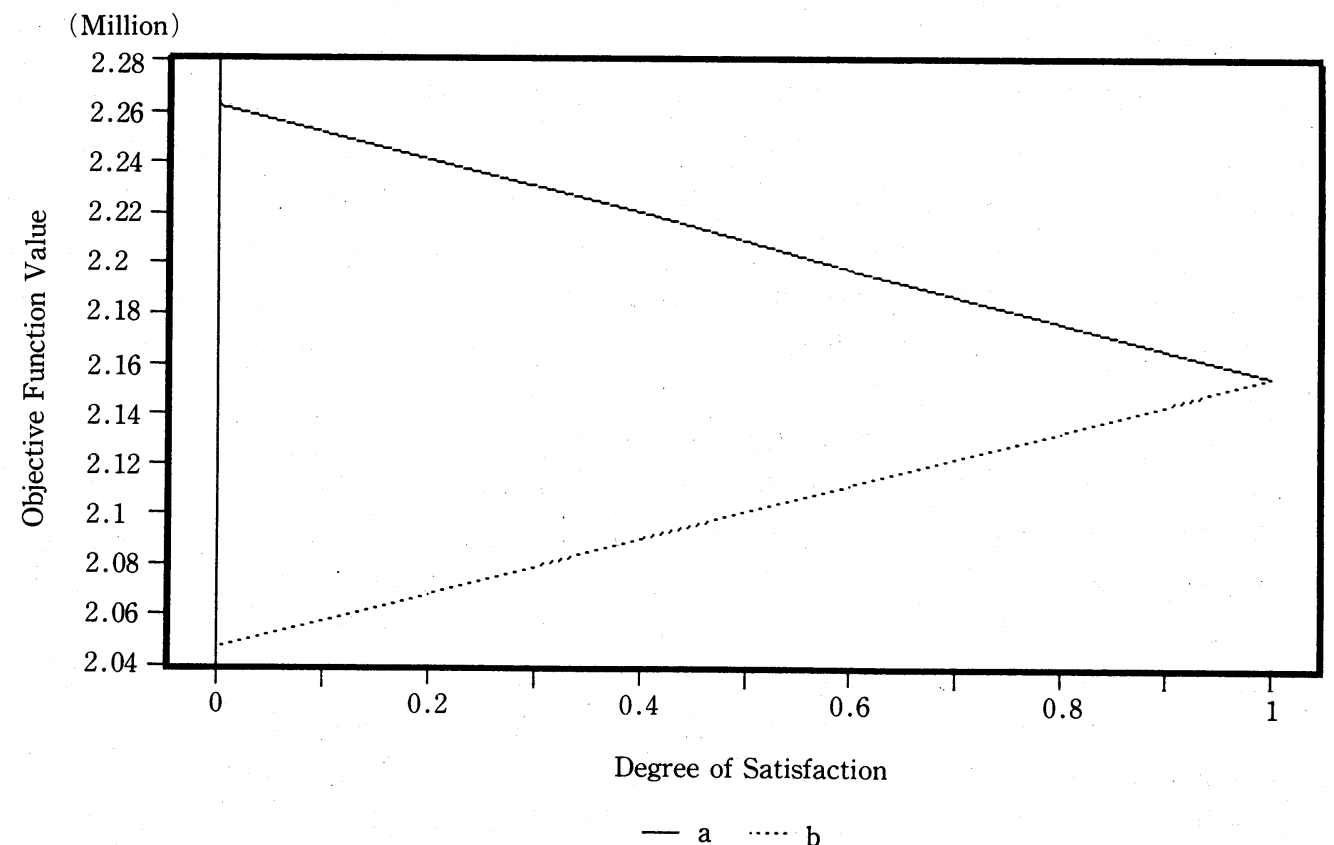

Fig. 6 Accessibility increase vrs degree of satisfaction in case III $\alpha$-inter-nondominated alternative (a) and $\alpha$-inter-dominating aternative (b)

Table 5 Accessibility increase vrs degree of satisfaction in case III

\begin{tabular}{c|c|c|c}
\hline \hline \multirow{2}{*}{$\begin{array}{c}\text { Degree of } \\
\text { Satisfaction }\end{array}$} & \multicolumn{2}{|c|}{$\begin{array}{c}\text { Objective Function Value } \\
\text { (Accessibility Increase) }\end{array}$} & Remarks \\
\cline { 2 - 4 } & $\mathrm{a}$ & $\mathrm{b}$ & $\begin{array}{c}\text { Change in Road } \\
\text { Selection }\end{array}$ \\
\hline 1.0 & 2154.734 & 2154.834 & No \\
0.8 & 2176.288 & 2133.098 & " \\
0.6 & 2197.876 & 2111.650 & " \\
0.4 & 2220.706 & 2090.105 & $"$ \\
0.2 & 2240.924 & 2068.560 & " \\
0.0 & 2262.476 & 2047.005 & \\
\hline
\end{tabular}

$\mathrm{a}=\alpha$-Inter-Nondominated Alternative

$\mathrm{b}=\alpha$-Inter-Dominating Alternative 
refers to $\alpha$-inter-nondominated and b refers to $\alpha$-inter-dominating. (See also Table 5).

From the results it can be determined whether or not $\tilde{g}$ is a real function. When the solution in both cases are the same then $\widetilde{g}$ is a real function. From our results, both $\alpha-$ inter-nondominated and $\alpha$-inter-dominating yield the same road selection, which indicates that our objective function which measures accessibility increase is a real function.

The roads selected in case III are the same as those in the crisp LP case.

\section{Summary of Findings and Conclusion}

Considering accessibility improvement (accessibility increase) as the main objective to be maximized in rural roads improvements decision making, this study has shown how LP models can effectively be applied to the selection of roads (allocation of financial resources). The results have indicated widespread allocation of budget among the roads as the optimum allocation. This is considered an interesting finding, since in conventional practice the available budget tends to be concentrated on few roads. Tracks have also been included in the optimal selection set contrary to conventional practice which tends to ignore them.

Employing flexible programming technique or FLP, it has been shown how the decision maker can play an important role in steering the road selection according to his degree of satisfaction and tolerance limit. This may have many practical implications, for instance, with the same budget if the aspiration level is reduced the decision maker can be completely satisfied even as a few feeder road links in the optimal selection set are replaced by tracks.

Similarly, with the same aspiration level if budget could be increased, some feeder road links could be replaced by tracks and DM would still be completely satisfied.

Taking cognizance of the fact that tracks are in more severely deteriorated conditions than feeder roads in the region, by the use of FLP, tracks can be favored in the selection and yet the decision maker can be completely satisfied. Since decision makers are generally politicians, the use of Flexible Programming will allow room for maneuvering in the allocation of resources for rural roads improvement.

It could be remarked that optimality in the case of fiexible programming means effectiveness contrasted to efficiency (in crisp LP). Fuzzy LP can obviously be considered as a technique for effective choice.

The use of Fuzzy numbers increases the effectiveness of applying FLP to the resource allocation problem in Developing countries. Many useful manipulation of the Fuzzy data available, using Inter, Incl and Incl $_{1}$ indexes can help throw more light on the road investment decision environment as illustrated in this study.

The use of FLP with fuzzy relation is yet another potentially useful tool to assist in improving the modeling of the objective function, which is the main criterion used in selecting the roads. It has indicated that our modeling of accessibility increase via time savings is very realistic.

The significance of Fuzzy Linear Optimization techniques to rural roads investment, as 
illustrated in this study, cannot be overemphasized. Using the fuzzy approach we can also handle multiple criteria decision situations (see for instance [8]). Our future research effort will be extended to the application of fuzzy multiple criteria decision analysis to rural roads investment decision making.

\section{References}

[1] Bellman, R.E. and L.A. Zadeh, "Decision-Making in a Fuzzy Environment", Management Science, Vol. 17, No. 4, December, 1970.

[2] Dubois, D., and H. Prade, Fuzzy Sets and Systems: Theory and Applications, Academic Press, 1980.

[ 3 ] Ecker, Joseph, G. and Michael Kupferschmid, Introduction to Operations Research, John Wiley and Sons, 1988.

[ 4 ] Howe, John, "Criteria for Road Planning", in Howe, John and Peter Richards, Eds., Rural Roads and Poverty Alleviation, Intermediate Technology Publications, 1984, pp. 18-47.

[5] Luhandjula, M.K., "Fuzzy Optimization: An Appraisal " Fuzzy Sets and Systems, 30 (1989), 257282.

[6] Mensa-Bonsu, I.F., Economic Appraisal of a Feeder Road Project : A case Study of the Proposed Sewia-Junction-Akwaduo-Jachi Feeder Road Improvement Project, B.Sc. Thesis, University of Science and Technology, Ghana, 1986.

[ 7 ] Mensa-Bousu, I.F., Optimum Resource Allocation for Rural Roads Improvement by Accessibility Maximizing, M.Sc. Thesis, Graduate School of Environmental Science, Hokkaido University, 1991.

[8] Seo, Fumiko and M. Sakawa, Multiple Criteria Decision in Regional Planning, D. Reidel Publishing Company, 1988.

[ 9 ] Shefer, D. and L. Kaess, "Evaluation Methods in Regional Planning", Town Planning Review, Vol. 61, No. 1, January, 1990.

[10] Sinha, K.C., Tien F. Fwa, and I. M. Mouaket, "New Tools and Techniques for Highway Maintenance Management", Transportation Research Record, No. 1276, 1990, pp. 28-36.

[11] Tanaka, H. and K. Asai, "Fuzzy Linear Programming with Fuzzy Numbers", Fuzzy Sets and Systems, 13 1984, pp. 1-10.

[12] Whalen, Thomas, "Introduction to Decision Making under various Kinds of Uncertainty" in Kacprzyk, J. and S.A. Orlovski (Eds.), Optimization Models Using Fuzzy Sets and Possibility Theory, D. Reidel Publishing Company, 1987.

[13] Wierzchon, S.T., "Linear Programming with Fuzzy Sets: A General Approach", Mathematical Modelling, Vol. 9, No.6, 1987, pp. 447-459.

[14] World Bank, Road Deterioration in Developing Countries, The World Bank 1988.

[15] Zadeh, L.A., "Fuzzy Sets as a Basis for a Theory of Possibility", Fuzzy Sets and Systems, 1 1978, pp. 3-28.

[16] Zimmermann, Hans J., Fuzzy Sets, Decision Making, and Expert Systems, Kluwer Academic Publishers, 1987. 